\section{Military Technical College Kobry El-kobbah, Cairo, Egypt}

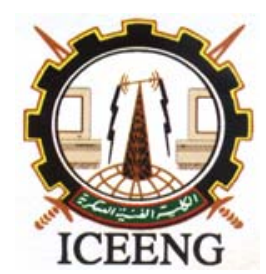

\title{
SUBOPTIMAL DATA ASSOCIATION TECHNIQUE FOR MULTIPLE-TARGET TRACKING IN DENSE CLUTTER ENVIRONMENT
}

\author{
Kamel $^{*}$ H. and Badawy ${ }^{* *}$ W.
}

\begin{abstract}
In multiple target tracking (MTT) systems that track targets with less-than-unity probability of detection in the presence of false alarms (FA), data association is very important. Data association is responsible for deciding which of the received multiple measurements should update which track. Some data association techniques use a unique pairing to update a track; i.e. at most one observation is used to update a track. An alternative approach is to use all of the validated measurements with different weights (probabilities), known as probabilistic data association (PDA). Due to the increase in the FA rate or low probability of target detection, most of the data association algorithms begin to fail. In this paper, we introduce a new suboptimal PDA technique for MTT in dense clutter environment. The proposed technique is based on merging the probabilistic nearest-neighbor filter (PNNF) with the PDA algorithm. The main idea is based on high-weighting the measurements that has minimum statistical distance from the predicted position of the target. The state updating equation in Kalman filter uses the combined innovation as in Joint Probabilistic Data Association method which is defined as the weighted sum of the residuals associated with many observations. Due to its simplicity in calculations and robustness, this technique can be used for real-time applications even though in dense clutter environments. We applied the proposed algorithm in tracking multiple targets in presence of various clutter densities. Results showed better performance when compared to Nearest-Neighbor and All-Neighbors approaches in different clutter densities and noise measurements.
\end{abstract}

\section{KEYWORDS}

Multiple target tracking, data association, probabilistic data association algorithm.

\footnotetext{
${ }^{*}$ Ph.D. Candidate, Elec. \& Comp. Eng. Department, University of Calgary, AB, Canada.

${ }^{* *}$ Associate Professor, Elec. \& Comp. Eng. Department, University of Calgary, AB, Canada.
} 


\section{INTRODUCTION}

In surveillance systems, target tracking is an essential requirement. Measurements from diverse sources (targets of interest, clutter, and/or internal thermal noise) are reported by sensors; e.g., radar, sonar, and infrared (IR) sensors. The main objective of a tracking system is to collect sensor data and classify them into sets of observations, or tracks that are produced by the same object (or target). In general, observations can be received at regular intervals of time (scan periods). Data association is the subject that deals with the integration of measurements (observations) from one or more sensors in multitarget tracking (MTT) systems. Each measurement may originate from one out-of-several target or from false detection (clutter and/or noise). In addition, a target may fail to be detected in some scans. The uncertainty in measurement origin is a complicated factor, in addition to the statistical in the values of measurements. In order to estimate the states of the targets, one needs to resolve this uncertainty, i.e. to associate with each measurement a unique target or to declare the measurement as clutter. If an incorrect observation is associated with a track, this track may diverge and causes other tracks to also diverge. Thus, data association is considered the crucial element and the most important component of any MTT system.

A number of algorithms have been developed to solve this problem [1,2], e.g., the strongestneighbor filter (SNF) and the nearest-neighbor filter (NNF). In the SNF, we use the signal with the highest intensity among the received measurements within a gate for track update and the others are discarded. Meanwhile, in the NNF, the measurement with the minimum statistical distance to the predicted measurement is used. Unfortunately, these approaches begin to fail as the FA rate increases or with low observable (low probability of target detection) targets $[3,4]$. An alternative approach - known as probabilistic data association (PDA) [5] - uses all of the validated measurements with different weights (probabilities). The standard PDA and its extensions have been shown to be very effective in tracking a single target in clutter $[4,6]$.

In case of tracking multiple targets, data association becomes more difficult because one measurement can be validated by multiple tracks in addition to a track validating multiple measurements as in the single-target case. To solve this problem, joint PDA (JPDA) algorithm is used to track multiple targets by evaluating the measurement-to-track association probabilities and combining them to find the state estimate [4]. A more powerful algorithm is the multiple hypothesis tracking (MHT). It handles the multitarget tracking problem by evaluating the likelihood that there is a target given a sequence of measurements. Although the MHT can be considered as an attempt to obtain an optima solution, its practical use in large scale problems is limited since it requires the evaluation of an exponentially increasing number of feasible joint association hypotheses [2]. In the tracking benchmark problem [7] designed to compare the performance of different algorithms for tracking highly maneuvering targets in the presence of electronic countermeasures, the PDA-based estimator, in conjunction with the interacting multiple model (IMM) estimator, yielded better performance compared to that of the MHT algorithm [7].

In this paper, we propose a new extension for the PDA capable for tracking multiple targets in dense clutter environment. The proposed technique is based on merging the nearest-neighbor filter (NNF) together with the PDA algorithm. The main idea is based on high-weighting the measurements that has minimum statistical distance from the predicted position of the target. The state updating equation in Kalman filter uses the combined innovation as in Joint Probabilistic Data Association method which is defined as the weighted sum of the residuals associated with many observations. The merged PDA (MPDA) algorithm is considered to be a simpler approach for MTT purposes in dense clutter environment. 
This paper is organized as follows. Section 2 presents an overview on the nearest-neighbor filter NNF and the Porbabilistic NNF (PNNF). In Section 3, a brief introduction to the basic PDA is given. The MPDA algorithm is defined in Section 4. Finally, performance analysis and simulation results are shown in Section 5 followed by a conclusion.

\section{NEAREST-NEIGHBOR APPROACH}

For its computational simplicity, the nearest-neighbor filter (NNF) is widely used for tracking a single target in a clutter environment. In the update step, together with the Kalman filter, the NNF uses the measurement with the least statistical distance from the predicted position as if it were target-originated. The main drawback of the NNF is the false alarms. To improve the performance of the NNF, the probabilistic NNF (PNNF) is introduced [9]. Three events are taken into account for the derivation of the estimation error covariance of the target in the PNNF: (1) the measurement is originated from the true target $\left(M_{T}\right),(2)$ the measurement is originated from a false target $\left(M_{F}\right)$; e.g. clutter, (3) there is no validated measurement at all $\left(M_{0}\right)$. The PNNF puts into consideration the probability of the event that the selected measurement is originated from the true target.

\subsection{Nearest-Neighbor Filter (NNF) Algorithm}

The NNF algorithm is driven in two steps: (1) prediction step identical to that used in Kalman filter and (2) update step as follows [8]:

(a) For the case of $M_{0}$

$$
\begin{aligned}
& \hat{x}_{k}=\bar{x}_{k} \\
& \hat{P}_{k}=\bar{P}_{k}+\frac{P_{D} P_{G}\left(1-C_{\tau_{g}}\right)}{1-P_{D} P_{G}} K_{k} S_{k} K_{k}^{T}
\end{aligned}
$$

(b) For the case of $M_{T}$ and $M_{F}\left(\bar{M}_{0}\right)$

$$
\begin{aligned}
& \hat{x}_{k}=\bar{x}_{k}+K_{k}\left(z_{k}-H_{k} \bar{x}_{k}\right) \\
& \hat{P}_{k}=\bar{P}_{k}-K_{k} S_{k} K_{k}^{T}
\end{aligned}
$$

where

$P_{D} \ldots$ probability of detection,

$P_{G} \ldots$ probability of the target within the validation gate, and

$C_{\tau_{g}}$... constant covariance ratio.

$\mathrm{P}_{\mathrm{G}}$ and $C_{\tau_{g}}$ are defined by the following equations: 


$$
\begin{aligned}
P_{G} & =\frac{1}{2^{n / 2} \Gamma(n / 2) \int_{0}^{\gamma} q^{\frac{n}{2}-1} e^{\frac{-q}{2}} d q} \\
C_{\tau_{g}} & =\frac{\int_{0}^{\gamma} q^{\frac{n}{2}} e^{\frac{-q}{2}} d q}{n \int_{0}^{\gamma} q^{\frac{n}{2}-1} e^{\frac{-q}{2}} d q}
\end{aligned}
$$

where $\sqrt{\gamma}$ is the gate size, $\Gamma(n / 2)=\left(2 \pi^{n / 2}\right) / n c_{n}$, and $c_{2}=\pi$ for $n=2$ in a 2-dimensional space. The target of interest is assumed to be detectable.

\subsection{Probabilistic Nearest-Neighbor Filter (PNNF) Algorithm}

The PNNF was introduced in [9] to overcome the drawback of the NNF. It takes into account the probabilities of the possible events engendered by the data association with the nearestneighbor measurement $\left\{M_{T}, M_{F}, M_{0}\right\}$. In this paper, we are going to use the PNNF as derived by Lee and Song in [8] under the assumption that the target is visible. The PNNF is summarized in two identical steps to that of the NNF algorithm:

(a) For the case of $M_{0}$

$$
\begin{aligned}
& \hat{x}_{k}=\bar{x}_{k} \\
& \hat{P}_{k}=\bar{P}_{k}+\frac{P_{D} P_{G}\left(1-C_{\tau_{g}}\right)}{1-P_{D} P_{G}} K_{k} S_{k} K_{k}^{T} \\
& S_{k}=H_{k} \bar{P}_{k} H_{k}^{T}+R_{k}
\end{aligned}
$$

(b) For the case of $\bar{M}_{0}$

$$
\begin{aligned}
& \hat{x}_{k}=\bar{x}_{k}-K_{k} \beta_{1} v_{k} \\
& \bar{P}_{k}^{M_{F}}=\bar{P}_{k}-K_{k} S_{k} K_{k}^{T}+\alpha K_{k} S_{k} K_{k}^{T} \\
& \hat{P}_{k}=\bar{P}_{k}^{M_{F}} \beta_{0}+\left(\bar{P}_{k}-K_{k} S_{k} K_{k}^{T}\right) \beta_{1}+\beta_{0} \beta_{1} K_{k} v_{k} v_{k}^{T} K_{k}^{T}
\end{aligned}
$$

where $\bar{P}_{k}^{M_{F}}$ is the update error covariance conditioned on $M_{F}, v_{k}$ is the residual of the NN measurement, and $\alpha$ and $\beta_{1}$ are given by:

$$
\begin{aligned}
& \alpha=\frac{1-P_{D} P_{R}(D) C_{\tau}(D)}{1-P_{D} P_{R}(D)} \\
& \beta_{1}=\frac{P_{D} e^{-\frac{n}{2}}}{P_{D} e^{-\frac{n}{2}}+(2 \pi)^{\frac{n}{2}}\left|S_{k}\right|^{\frac{1}{2}} \lambda\left(1-P_{D} P_{R}(D)\right)}
\end{aligned}
$$


where $n=2$ for 2-dimensional space, $D$ is the normalized distance squared (NDS) of the NN measurement, $\lambda$ is the spatial clutter density, $\beta_{0}$ is the probability that the NN measurement is not target originated $\left(\beta_{0}=1-\beta_{1}\right)$, and $P_{R}(D)$ is the probability of the target within an elliptic gate with gate size $\sqrt{D}$ and $C_{\tau}(D)$ are given by the following equations:

$$
\begin{aligned}
P_{R}(D) & =\frac{1}{2^{\frac{n}{2}} \Gamma(n / 2)} \int_{0}^{D} q^{\frac{n}{2}} e^{-\frac{q}{2}} d q \\
C_{\tau}(D) & =\frac{\int_{0}^{D} q^{\frac{n}{2}} e^{-\frac{q}{2}} d q}{n \int_{0}^{D} q^{\frac{n}{2}-1} e^{-\frac{q}{2}} d q}
\end{aligned}
$$

\section{3- THE PROBABILISTIC DATA ASSOCIATION (PDA) ALGORITHM}

The Probabilistic Data Association (PDA) [10] is considered as the first technique introduced based on the all-neighbors (AN) approach. However, data association becomes more difficult with multiple targets. Here, a measurement itself can be validated by multiple tracks. Thus a modified technique denoted Joint PDA (JPDA) [11] was derived to include the presence of other observations coming from other targets by evaluating the measurement-to-track association probabilities and combining them to find the state estimate. To overcome the computational complexity of the JPDA, several algorithms was generated, e.g., Ad hoc JPDA [12], suboptimal JPDA [13], near-optimal JPDA [14], integrated PDA [15], and dominant PDA [16].

In the tracking benchmark problem designed to compare the performance of different algorithms for tracking highly-maneuverable targets in the presence of electronic countermeasures, the PDA-based estimator, in conjunction with Interacting Multiple Models (IMM), showed better performance when compared to that of the Multiple Hypothesis Tracking (MHT), which handles the MTT problem by evaluating the likelihood that there is a target giving a sequence of measurements [2]. However, the IMMPDA combines two PDA algorithms and has a larger window than PDA. Therefore, it results in more computational complexity than PDA. Meanwhile, comparing PDA to other data association techniques, e.g., Viterbi Data Association (VDA) and Fuzzy Data Association (FDA) techniques, showed the failure of PDA to track targets in low Signal-to-Noise Ratio (SNR) [17]. On the other hand, PDA has many other applications in many tracking scenarios, e.g., tracking low observable targets in passive sonar measurements and tracking the state of maneuvering target using measurement from an electrooptical sensor.

\subsection{Assumptions}

The PDA algorithm calculates in real-time the probability that each validated measurement is assignable to the target of interest. Assuming that there is a unique target of interest whose state evolves according to a dynamic equation driven by process noise, the state of the target of interest, of dimension $n_{x}$, is assumed to evolve in time according to:

$$
X_{k+1}=F_{k} X_{k}+G_{k} W_{k}
$$


where $X_{k}$ is n-dimensional state vector at $k^{\text {th }}$ stage, $F_{k}$ is $\mathrm{n} \times \mathrm{n}$ transition matrix, $G_{k}$ is $\mathrm{n} \times 1$ transition matrix, and $W_{k}$ is $n$-dimensional zero-mean white Gaussian noise with know covariance matrix $Q_{k}$. The measurement model, of dimension $m_{z}$, is described as follows:

$$
Z(k)= \begin{cases}H_{k} X_{k}+V_{k} & \text { target case } \\ C_{k} & \text { clutter case }\end{cases}
$$

where $Z_{k}$ is $m$-dimensional measurement vector, $H_{k}$ is $m \times n$ observation matrix, and $V_{k}$ is $m$ dimensional measurement zero-mean white Gaussian noise mutually independent with $W_{k}$ with known covariance matrix $R_{k}$. As the measurements are in polar coordinates and tracking algorithm is done in Cartesian coordinates, the measurements are coupled. The covariance matrix of the measurement noise $V_{k}$ may be written as:

$$
R_{k}=\left[\begin{array}{cc}
\sigma_{x, k}^{2} & \sigma_{x y, k}^{2} \\
\sigma_{y x, k}^{2} & \sigma_{y, k}^{2}
\end{array}\right]
$$

which is given by:

$$
R(k)=A_{2} R_{0} A_{2}^{T}
$$

where:

$$
\begin{aligned}
A_{2} & =\left[\begin{array}{cc}
\cos (\theta) & -\sin (\theta) \\
\sin (\theta) & \cos (\theta)
\end{array}\right] \\
R_{0} & =\left[\begin{array}{cc}
\sigma_{r}^{2} & 0 \\
0 & r^{2} \sigma_{\theta}^{2}
\end{array}\right]=\left[\begin{array}{cc}
\sigma_{x}^{2} & 0 \\
0 & \sigma_{y}^{2}
\end{array}\right]
\end{aligned}
$$

where $A_{2}$ is the rotational matrix, $\sigma_{r}^{2}$ and $\sigma_{\theta}^{2}$ are the range and azimuth measurement noise respectively. We clarified this part in equation The number of clutter observations $C(k)$ are assumed to have Normal distribution and their locations are assumed to have the uniform distribution on the surveillance region [1,2]. The past information about the target is approximated to be:

$$
p\left[x_{k} \mid Z^{k-1}\right]=N\left[x_{k} ; \hat{x}_{k}, \hat{P}_{k}\right]
$$

where $N\left[x_{k} ; \bar{x}_{k}, \bar{P}_{k 1}\right]$ denotes the normal probability density function (pdf) with argument $x_{k}$, $\bar{x}_{k}$ mean and $\bar{P}_{k}$ covariance matrix. At each time, a validation region is set up as in (16). At most one of several validated measurements can be target-originated if the target was detected and the corresponding measurement fell into the validation gate. The remaining measurements are assumed to be FAs or clutter and are modeled as independent identically distributed (i.i.d.) measurements with uniform spatial distribution. The target detections occur independently over time with known probability $P_{D}$. These assumptions enable a state 
estimation scheme to be obtained, which is almost as simple as the Kalman filter (KF), but much more effective in clutter.

\subsection{Probabilistic Data Association Filter (PDAF)}

The PDAF uses a decomposition of the estimation with respect to the origin of each element of the latest set of validated measurements, denoted as:

$$
Z_{k}=\left\{z_{k}^{i}\right\}_{i=1}^{m_{k}}
$$

where $\left\{z_{k}^{i}\right\}_{i=1}^{m_{k}}$ is the $i^{\text {th }}$ validated measurement and $m_{k}$ is the number of measurements in the validation region at time $k$. The cumulative set (sequence) of measurements is:

$$
Z^{k}=\{Z(j)\}_{j=1}^{k}
$$

\subsection{Measurement Validation}

From the Gaussian assumption driven in (13), the validation region is the elliptical region

$$
\Lambda_{k, \sqrt{\gamma}}=\left\{z:\left[z-\bar{z}_{k}\right]^{T} S_{k}\left[z-\hat{z}_{k}\right] \leq \sqrt{\gamma}\right\}
$$

where $\sqrt{\gamma}$ is the gate size and $S_{k}$ is covariance of innovation corresponding to the true measurement defined as in (4). The area of the validation region is

$$
\Lambda_{k}=c_{m_{z}}\left|\sqrt{\gamma} S_{k}\right|^{1 / 2}=c_{m_{z}} \gamma^{\frac{1}{4}}\left|S_{k}\right|^{\frac{1}{2}}
$$

where $c_{m_{z}}=c_{2}=\pi$ as we are evaluating a 2-dimensional space.

\subsection{State Estimation}

According to the assumptions, the association events

$$
\eta_{i}(k)=\left\{\begin{array}{cc}
z_{k}^{i} \text { is the target }- \text { originated measurement } & i=1, \ldots, m_{k} \\
\text { clutter } & i=0
\end{array}\right.
$$

Are mutually exclusive for $m_{k} \geq 1$. The estimate conditioned on measurement $i$ being correct is

$$
\bar{x}_{i, k}=\hat{x}_{k}+W_{k} v_{i, k} \quad i=1, \ldots, m_{k}
$$

where the corresponding innovation is

$$
v_{i, k}=z_{i, k}-\hat{z}_{k}
$$

and the gain $W_{k}$ is the identical to that in Kalman filter

$$
W_{k}=\hat{P}_{k} H_{k}^{T} S_{k}^{-1}
$$

In case of clutter or no measurement are within the gate area, the track will be updated using the predicted position, i.e.,

$$
\bar{x}_{0, k}=\hat{x}_{k}
$$

\subsection{State and Covariance Update}

Combining (19) and (22), we get the state update equation in the PDAF:

$$
\bar{x}_{k}=\hat{x}_{k}+W_{k} v_{k}
$$


where the combined innovation is:

$$
v_{k}=\sum_{i=1}^{m_{k}} \beta_{i, k} v_{i, k}
$$

where $\beta_{i, k}=P\left\{\eta_{i, k} \mid Z^{k}\right\}$ is the conditional probability of an event.

\subsection{Prediction Equations}

The prediction of the state and measurement to scan period $k+1$ as done as in the standard Kalman filter:

$$
\begin{aligned}
& \hat{x}_{k+1}=F_{k} \bar{x}_{k} \\
& \hat{z}_{k+1}=H_{k+1} \hat{x}_{k+1}
\end{aligned}
$$

The covariance of the predicted state is driven similarly:

$$
\hat{P}_{k+1}=F_{k} \bar{P}_{k} F_{k}^{T}+Q_{k}
$$

Our main objective in this paper is to prove the ability of the PDA - when merged with PNNF algorithm - to track multiple targets even though in dense clutter environment, e.g. low SNR.

\section{MERGED PROBABILISTIC DATA ASSOCIATION (MPDA)}

In this section, we introduce a new suboptimal PDA algorithm. The main idea lies on merging both PNNF together with the PDA algorithm. It is mainly based on the weighted sum of all observations within the validation gate by giving a probability weight to each observation. This probability weight depends on measuring the statistical distance from the predicted target position and each observation within its validation gate as defined in the PNNF approach in (7).

Assume at a scan period $k$, we have a predicted position of the target of interest $\hat{x}_{k+1}$ given by:

$$
\hat{x}_{k}=F_{k-1} \bar{x}_{k-1}
$$

which lies within an elliptical area validation gate with size $\sqrt{\gamma}$ given by (17):

$$
\Lambda_{k}=c_{m_{z}}\left|\sqrt{\gamma} S_{k}\right|^{1 / 2}=c_{m_{z}} \gamma^{\frac{1}{4}}\left|S_{k}\right|^{\frac{1}{2}}
$$

where $S_{k}=H_{k} \bar{P}_{k} H_{k}^{T}+R_{k}$ and $c_{m_{z}}=c_{2}=\pi$ for 2-dimensional domain. Consider the situation that there exist $N$ observations within the validation gate of the track $i$. These received observations may not all arise from the target of interest; some of them may be extraneous returns like clutter, noise, or false alarms. Consequently, we take into account the probabilities of the possible events engendered by the data association with the NN measurement $\left\{M_{T}, M_{F}, M_{0}\right\}$. Applying the PNNF together with the PDA algorithm, the update step will be as follows: 
(a) For the case of $M_{0}$

$$
\begin{aligned}
& \hat{x}_{k}=\bar{x}_{k} \\
& \hat{P}_{k}=\bar{P}_{k}+\frac{P_{D} P_{G}\left(1-C_{\tau_{g}}\right)}{1-P_{D} P_{G}} K_{k} S_{k} K_{k}^{T} \\
& S_{k}=H_{k} \bar{P}_{k} H_{k}^{T}+R_{k}
\end{aligned}
$$

(b) For the case of $\bar{M}_{0}$

$$
\begin{array}{rlr}
\hat{x}_{k} & =\bar{x}_{k}+W_{k} v_{k} \\
v_{k} & =\sum_{i=1}^{N} \beta_{i, k} v_{i, k} & \\
\beta_{i, k} & =P\left\{\eta_{i, k} \mid Z^{k}, N, Z^{k-1}\right\} \quad i=1, \ldots, N & \\
& =\frac{1}{c} p\left[Z_{k} \mid \eta_{i, k}, N, Z^{k-1}\right] \times P\left\{\eta_{i, k} \mid N, Z^{k-1}\right\} & \\
p\left[Z_{k} \mid \eta_{i, k}, N, Z^{k-1}\right]=V_{k}^{-N+1} P_{G}^{-1}\left[v_{i, k}, 0, S_{k}\right] & i=1, \ldots, N \\
\bar{P}_{k}^{M_{F}} & =\bar{P}_{k}-K_{k} S_{k} K_{k}^{T}+\alpha K_{k} S_{k} K_{k}^{T} & \\
\hat{P}_{k} & =\bar{P}_{k}^{M_{F}} \beta_{0}+\left(\bar{P}_{k}-K_{k} S_{k} K_{k}^{T}\right) \beta_{1}+\beta_{0} \beta_{1} K_{k} v_{k} v_{k}^{T} K_{k}^{T} & \\
v_{i, k} & =z_{i, k}-\bar{z}_{k}=z_{i, k}-H_{k} \hat{x}_{k} &
\end{array}
$$

with residual covariance matrix defined by:

$$
S_{i, k}=H_{k} \bar{P}_{k} H_{k}^{T}+R_{k}
$$

The probability of track $j$ being associated with observation $i$ within the validation gate is defined by:

$$
P_{G}=\left\{\begin{array}{cc}
0 & i=0 \text { (no valid observation }) \\
\frac{1}{2^{\frac{n}{2}} \Gamma(n / 2)} \int_{0}^{D} q^{\frac{n}{2}} e^{-\frac{q}{2}} d q & 1 \leq i \leq N
\end{array}\right.
$$

Where $D$ is the statistical distance measured between the $j^{\text {th }}$ observation and track $i$ which is given by:

$$
D=\sqrt{\left(\hat{x}_{i}-x_{j}\right)^{2}+\left(\hat{y}_{i}-y_{j}\right)^{2}}
$$

The formula mentioned in (32) to calculate $P_{G}$ gives a high weighting to the measurement that has a minimum statistical distance from the predicted position of the target. Furthermore, this weight decreases as a measurement position far from the predicted position of the target. Moreover, this weight is evaluated on a probabilistic basis according to which event we have. After computing the probabilities using equation (32), the state updating equation in Kalman 
filter uses the combined innovation as in PDA method which is defined as the weighted sum of the residuals associated with $m$ observations mentioned in (24) as $v_{k}=\sum_{i=1}^{m_{k}} \beta_{i, k} v_{i, k}$.

\section{PERFORMANCE ANALYSIS}

The number of clutter observations is assumed to be Gaussian distributed. The noise is stochastic independent on the target trajectories. Fig. 1 shows an example of clutter observations surrounding target plots. The location of clutter observations is assumed to have a uniform distribution around the predicted target position.

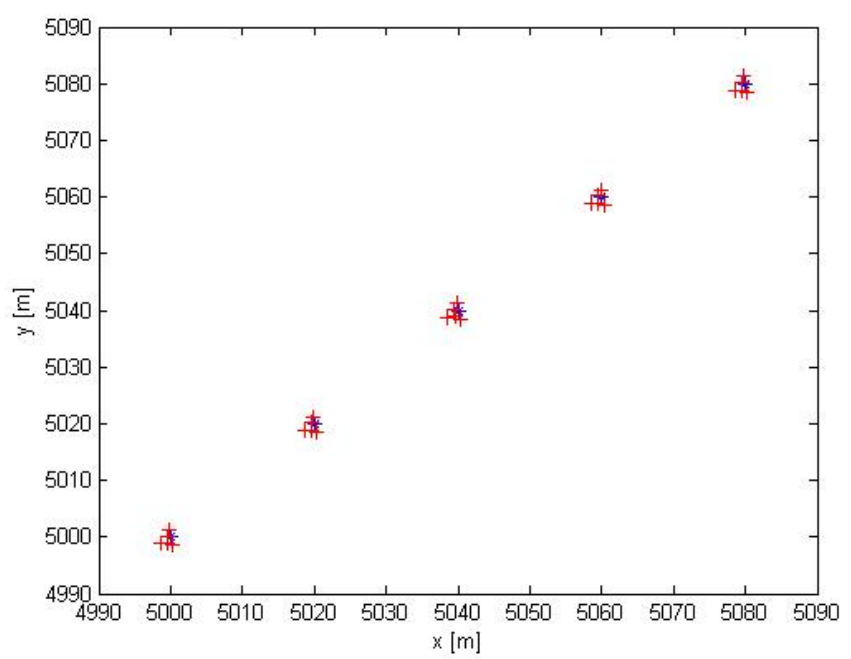

Fig. 1 Example on clutter surrounding targets with $b=0.03$

The target motion is modeled in two dimensions $x-y$ coordinates. The time invariant $F$ and $G$ matrices are given by :

$$
F=\left[\begin{array}{llll}
1 & 0 & T & 0 \\
0 & 1 & 0 & T \\
0 & 0 & 1 & 0 \\
0 & 0 & 0 & 1
\end{array}\right] \quad G=\left[\begin{array}{cc}
T^{2} / 2 & 0 \\
0 & T^{2} / 2 \\
T & 0 \\
0 & T
\end{array}\right]
$$

The state matrix $H$ is defined by :

$$
H=\left[\begin{array}{llll}
1 & 0 & 0 & 0 \\
0 & 1 & 0 & 0
\end{array}\right]
$$

The measurement noise $V_{k}$ is defined as zero-mean white Gaussian noise and covariance matrix $R$ :

$$
R=\left[\begin{array}{cc}
\sigma_{x}^{2} & 0 \\
0 & \sigma_{y}^{2}
\end{array}\right]=\left[\begin{array}{cc}
\sigma_{r}^{2} & 0 \\
0 & r^{2} \sigma_{\theta}^{2}
\end{array}\right]
$$


where $\sigma_{x}$ and $\sigma_{y}$ are the standard deviations of the measurement noise in both $x$ and $y$ directions, respectively.

Fig. 2 shows the error performance in tracking a single target using NNF with minimum distance, NNF with normalized distance, JPDA, and our proposed algorithm. It is clear that our proposed algorithm has better convergence to the steady-state error as well as the least mean-square-error value.

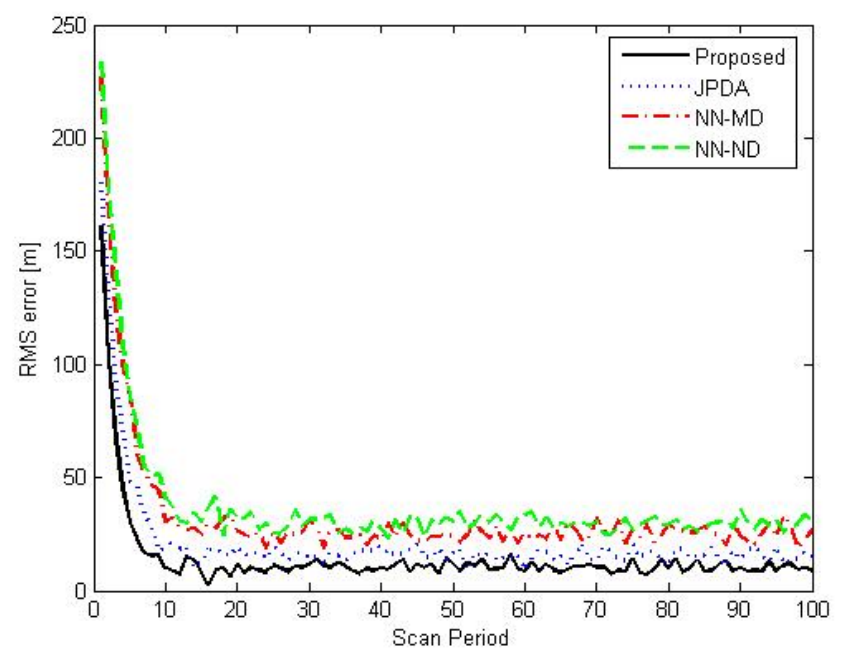

Fig. 2 The ensemble error performance in tracking a single target

Figure 3 represents the error in position between the true and estimated trajectories. We can notice that the maximum error does not exceed $450 \mathrm{~m}$ in the early scan periods. Then, the error converges to its minimum value and reaches the minimum value by the $20^{\text {th }}$ scan period aproximately.

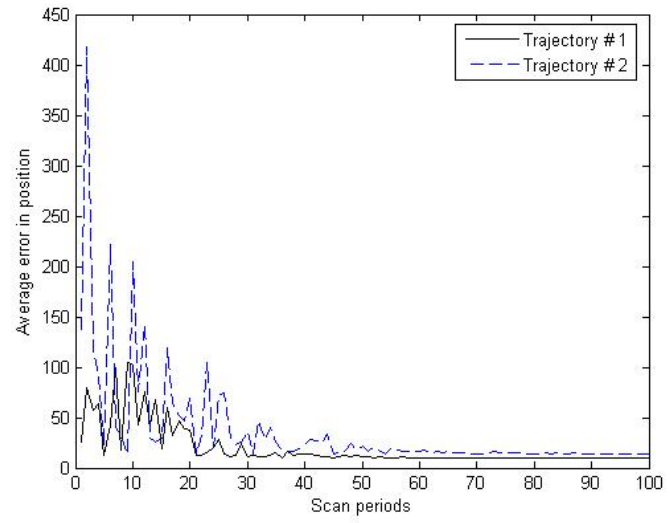

Fig. 3 Error in position between true and estimated trajectories.

To show the performance of the MPDA, we addrress the problem of tracking the scenario of two small-angle crossing targets. Fig. 4 shows the first scenario. For comparison purpose, we tracked the same scenario using (a) JPDA algorithm and (b) the proposed MPDA algorithm. As shown, the JPDA failed to track two small-angle crossing targets and one track is lost. Meanwhile, the proposed technique is quite successful in tracking both targets. The high 
convergence speed as well as the steady-state error of the proposed algorithm is shown in Figure 5 for both tracks.

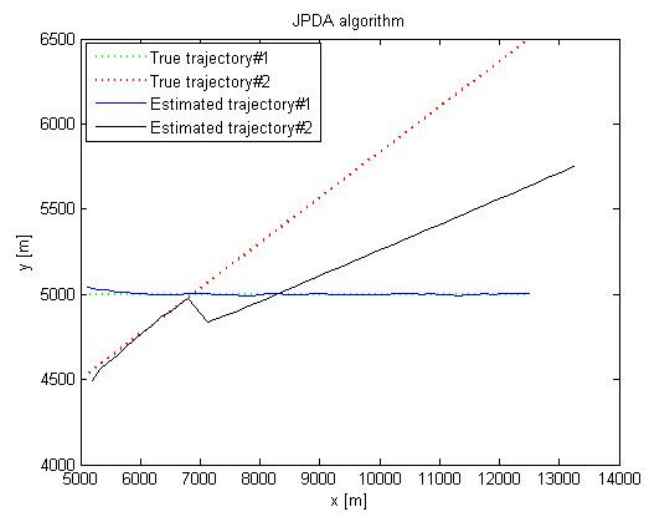

(a)

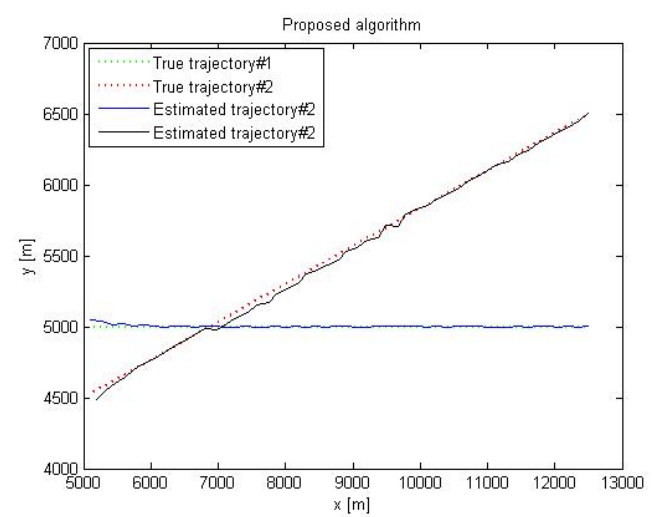

(b)

Fig. 4 True and predicted trajectories for two small-angle crossing targets immersed in dense clutter ( $b=0.03$ ) using (a) JPDA and (b) MPDA techniques

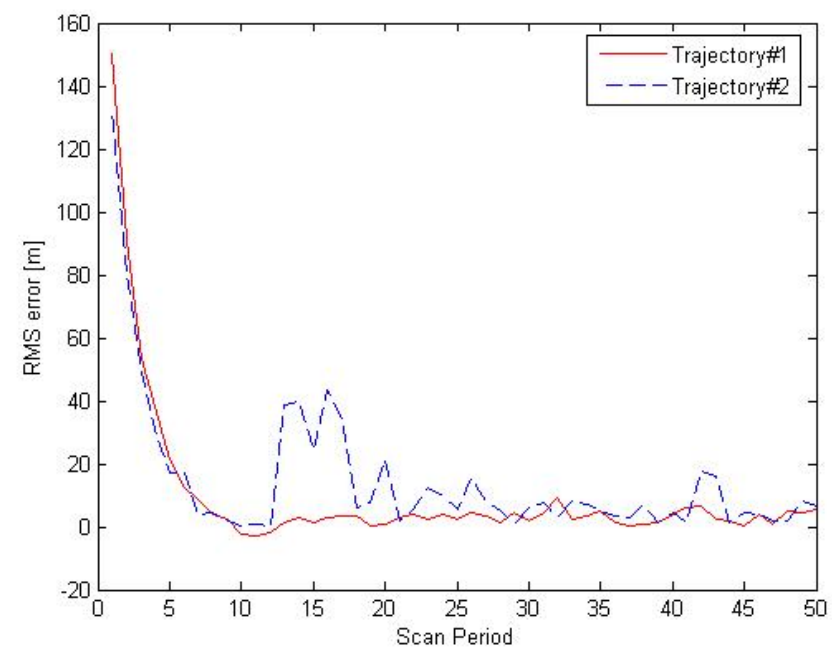

Fig. 5 RMSE between the estimated and true trajectories for small-angle crossing tracks using MPDA

\section{CONCLUSION}

In this paper, we introduced a new merged probabilistic data association (MPDA) technique to track multiple targets in various tracking situations. The MPDA was compared to other data association techniques. The results showed that the MPDA is more efficient than NNND, NN-MD, and PDA approaches in tracking difficult situations, e.g. low-angle crossing tracks and close parallel tracks in dense clutter environment and high noise measurements. Also, the MPDA has a relative high convergence speed. The main advantage of the MPDA over the IMMPDA, VDA, and FDA techniques is the simplicity. Due to the simplicity 
together with the high convergence speed, the MPDA is quite efficient for real-time applications.

\section{REFERENCES}

[1] Bar-Shalom, Y., Ed., Multitarget-Multisensor Tracking: Applications and Advances. Norwood, MA: Artech House, 1990, vol. I. Reprinted: Storrs, CT: YBS, 1998.

[2] Blackman, S. and Popoli, R., Design and Analysis of Modern Tracking Systems. Norwood, MA: Artech House, 1999.

[3] Dezert, J., "Improvement of strapdown inertial navigation using PDAF," IEEE Trans. Aerospace and Electronic Systems, vol. 35, pp. 835-856, July 1999.

[4] Kirubarajan, T., Bar-Shalom, Y., Blair, W. D., and Watson, G. A., "IMMPDA solution to benchmark for radar resource allocation and tracking in the presence of ECM," IEEE Transactions on Aerospace and Electronic Systems., vol. 34, pp. 1023-1036, Oct. 1998.

[5] Bar-Shalom, Y. and Li, X. R., Multitarget-Multisensor Tracking: Principles and Techniques. Storrs, CT: YBS, 1995.

[6] Lerro, D. and Bar-Shalom, Y., "Interacting multiple model tracking with target amplitude feature," IEEE Trans. Aerospace and Electronic Systems, vol. 29, pp. 494-509, Apr. 1993.

[7] Blair, W. D., Watson, G. A., Kirubarajan, T., and Bar-Shalom, Y. "Benchmark for radar resource allocation and tracking in the presence of ECM," IEEE Transactions on Aerospace and Electronic Systems, vol. 34, pp. 1015-1022, Oct. 1998.

[8] Lee, D.G. and Song, T.L., "Performance analysis of NNF-class target tracking algorithms applied to benchmark problem," The $5^{\text {th }}$ Asian Control Conference, Melbourne, Australia, July 20-23, 2004, pp. 1602-1607.

[9] Li, X.R., "The PDF of nearest-neighbor measurement and a probabilistic nearestneighbor filter for tracking in clutters," Proceedings of the $32^{\text {nd }} C D C$, San Antonio, Texas, Dec. 1993, pp. 918-923.

[10]Bar-Shalom, Y. and Tse, E., "Tracking in a Cluttered Environment with Probabilistic Data Association,” Automatica, vol. 11, September 1975, pp. 451-460.

[11]Fortemann, T., Bar-Shalom, Y., and Scheffe, M., "Multi-Target Tracking Using Joint Probabilistic Data Association," In Proceeding of IEEE Conference on Decision and Control, December 1980, pp.807-812.

[12]Fitzgerald, R., "Development of Practical PDA Logic for Multitarget Tracking by Microprocessor,” in Multi-Target Multi-Sensor Tracking: Advanced Application, edited by Y. Bar-Shalom, Artech House, Norwood, MA, 1990, pp. 1-23.

[13]Rocher, J. and Phillis, G., "Suboptimal Joint Probabilistic Data Association," IEEE Transactions on Aerospace and Electronic Systems, vol. 29, No. 2, April 1993, pp. 510517.

[14]Roeccker, A. and Loral, “A Class Near Optimal JPDA Algorithms,” IEEE Transactions on Aerospace and Electronic Systems, vol. 30, No. 2, April 1994, pp. 504-510.

[15]Musicki, D., Evans, R. and Stankovic, S., "Integrated Probabilistic Data Association,” IEEE Transactions on Automatic Control, vol. 39, No. 6, June 1994, pp. 1237-1241.

[16]Pan, Q. and Zang, H., "Multitarget Tracking Using Dominant Probability Data Association,” Proceedings of the American Control Conference, January 1994, pp. 10471050.

[17]Gad, A., Majdi, F. and Farooq, M., “A Comparison of Data Association Techniques for Target Tracking in Clutter," Proceedings of the $5^{\text {th }}$ International Conference on Information Fusion, 2002, vol. 2 , 8-11 July, 2002, pp.1126-1133. 
Proceedings of the $\mathbf{5}^{\text {th }}$ ICEENG Conference, 16-18 May, 2006 